\title{
Anormalidades durante el desarrollo embrionario de sardina común, Strangomera bentincki en el ambiente natural
}

\author{
Abnormalities during the embryonic development in araucanian herring, \\ Strangomera bentincki, in natural environment \\ Paulina Vásquez ${ }^{1,2}$, Alejandra Llanos-Rivera ${ }^{1,3}$ \\ y Leonardo Castro ${ }^{1,4}$
${ }^{1}$ Programa de Investigación Marina de Excelencia (PIMEX-Nueva Aldea), Facultad de Ciencias Naturales y Oceanográficas, Universidad de Concepción, Casilla 160-C, Concepción, Chile
${ }^{2}$ Programa de Magíster en Ciencias, mención Oceanografía, Facultad de Ciencias Naturales y Oceanográficas, Universidad de Concepción, Casilla 160-C, Concepción, Chile
${ }^{3}$ Unidad de Biotecnología Marina, Facultad de Ciencias Naturales y Oceanográficas, Universidad de Concepción, Casilla 160-C, Concepción, Chile
${ }^{4}$ Laboratorio de Oceanografía Pesquera y Ecología Larval, Centro FONDAP-COPAS y Departamento de Oceanografía, Facultad de Ciencias Naturales y Oceanográficas, Universidad de Concepción,Casilla 160-C, Concepción, Chile paulivasquez@udec.cl

\begin{abstract}
To describe and quantify morphological abnormalities during the embryonic development of Strangomera bentincki an area nearby the Itata River mouth was sampled during the reproductive season 2007. Abnormality levels (3-13\%) were coincident with reported levels for other Clupeids species inhabiting areas with similar environmental characteristics to the area studied. Type and abnormalities
\end{abstract}

prevalence were coincident with those reported for other fish species. Blastopore irregular closing and the embryonic axis twist were the most frequent in the samples. These abnormality types would be caused by errors during the epiboly, which can occur spontaneously, influenced by internal or external factors.

Key words: Frequency of abnormalities, malformations, fish eggs

\section{Introducción}

La transparencia de los huevos de un gran número de especies de teleósteos facilita la observación y el análisis del desarrollo embrionario, haciendo posible el examen de algunas de sus cuatro etapas fundamentales: fecundación, segmentación, gastrulación y organogénesis (Kunz 2004). Estas etapas claves para la formación del organismo destacan por la complejidad de sus procesos, presentándose en algunos casos, irregularidades reflejadas en anormalidades morfológicas en el embrión. Estas pueden ocurrir tempranamente como alteraciones en el patrón de segmentación de los blastómeros, afectando de manera adversa el desarrollo subsecuente del embrión (Avery et al. 2009), mientras que otras se manifiestan más tardíamente, como por ejemplo, la diferenciación anormal del eje dorsoventral (Stachel et al. 1993). Las alteraciones del desarrollo embrionario pueden ocurrir espontáneamente influenciadas por factores internos o pueden ser provocadas por factores externos como las características hidrográficas naturales que son críticas para el desarrollo normal de los huevos (temperatura, salinidad) y por la exposición a xenobióticos (De Ciechomski 1966, Cameron et al. 1992). Esto último ha llevado a la utilización del nivel de anormalidades como bioindicador del estado del ambiente marino (Kjorsvik et al. 1990, Von Westernhagen et al. 2001).

La primera fase necesaria para individualizar anormalidades es la identificación de la secuencia de estadios que componen el desarrollo embrionario de la especie en estudio. Dependiendo del autor, los criterios de clasificación pueden variar, incluso para especies del mismo género. En el género Engraulis, por ejemplo, para E. ringens hay una escala de cinco estadios (Fischer 1958) mientras que para E. mordax existe una de once estadios (Moser \& Ahlstrom 1985). Independiente de la escala utilizada, los estudios que han caracterizado anormalidades en distintas especies coinciden en que el periodo de la gastrulación es donde más incidencia de anormalidades se observa. Adicionalmente Von Westernhagen (1988) y Makhotin et al. (2001), mediante experimentos de incubación con embriones de Gadus 
morhua, muestran que las anormalidades producidas durante la gastrulación contribuyen mayormente a la mortalidad natural de los embriones. Estos experimentos también demuestran la correspondencia de este tipo de anormalidades con bajas tasas de eclosión y altas tasas de mortalidad larval. Lo anterior indicaría la repercusión de la alteración en la gastrulación en la viabilidad de los embriones y en consecuencia su eventual efecto sobre la capacidad reproductiva de una población determinada (Von Westernhagen 1988, Cameron et al. 1992). Los antecedentes aquí señalados resaltan la relevancia que adquiere la investigación de anormalidades en el desarrollo embrionario de peces, más aún si se trata de especies de importancia económica (Mellinger 1995).

Estudios que traten la incidencia de anormalidades en peces nativos son escasos. En este contexto y dadas sus características biológicas un buen modelo a estudiar es la sardina común, Strangomera bentincki (Norman, 1936). Esta especie nerítico costera se distribuye desde Coquimbo $\left(29^{\circ} \mathrm{S}\right)$ hasta la Península de Taitao $\left(46^{\circ} \mathrm{S}\right)$ en la costa chilena (Serra et al. 1979, Córdova \& Balbontín 2006) siendo fuertemente explotada (790.000 ton año ${ }^{-1}$, SERNAPESCA 2008). La sardina común posee un ciclo de vida corto, crecimiento rápido, una elevada tasa de mortalidad natural y una estación reproductiva que se extiende desde julio a septiembre (Cubillos et al. 1999, 2002). Sus huevos son planctónicos y, además de su transparencia, poseen características tales como un corion delgado, frágil y sin ornamentaciones, un espacio perivitelino muy reducido, vitelo segmentado, ausencia de gota oleosa y un diámetro de $0,96 \mathrm{~mm}$ aproximadamente (Herrera et al. 1987). Todos estos atributos facilitan su identificación en las muestras de plancton y la observación de posibles alteraciones del desarrollo. El desove se produce en áreas ubicadas a menos de 10 millas náuticas de la costa $(<100 \mathrm{~m}$ de profundidad), sobre la plataforma continental y en bahías que facilitan la retención y concentración de los huevos (Cubillos et al. 2007).

En la zona centro-sur de Chile, un foco importante de desove y crianza de esta especie se encuentra en zonas aledañas a la desembocadura del río Itata, con altas concentraciones de huevos en el plancton (50-100 huevos $0,05 \mathrm{~m}^{-2}$ ) (Cubillos et al. 1999), por lo que constituye un área ideal para evaluar el nivel de anormalidades de esta especie en condiciones naturales. El presente estudio describe y cuantifica las anormalidades morfológicas ocurridas a lo largo de la época reproductiva 2007 de $S$. bentincki en zonas aledañas a la desembocadura del Río Itata.

\section{Material y métodos}

El área de estudio correspondió a estaciones de muestreo ubicadas en áreas costeras adyacentes a la desembocadura del río Itata y a Punta Burca en la bahía de Coliumo, zona centro sur de Chile (Tabla 1). Se realizaron muestreos planctónicos durante la época reproductiva de

Tabla 1

Fecha de muestreo, ubicación geográfica, temperatura superficial, número de huevos analizados y porcentajes de anormalidades en huevos de Strangomera bentincki durante el año 2007

Sampling date, location, superficial temperature, number of analyzed egg and

Strangomera bentincki malformations egg rate during 2007 year

\begin{tabular}{|c|c|c|c|c|c|c|}
\hline Fecha & $\begin{array}{l}\text { Latitud } \\
\left({ }^{\circ} \mathrm{S}\right)\end{array}$ & $\begin{array}{l}\text { Longitud } \\
\qquad\left({ }^{\circ} \mathrm{W}\right)\end{array}$ & $\begin{array}{c}\text { Temperatura } \\
\left({ }^{\circ} \mathrm{C}\right)\end{array}$ & $\begin{array}{l}\mathrm{N}^{\circ} \text { embriones } \\
\text { analizados }\end{array}$ & $\begin{array}{c}\text { Anormales } \\
(\%)\end{array}$ & $\begin{array}{c}\text { Promedio ( } \pm \text { D.E.) } \\
\text { mensual anormalidades }\end{array}$ \\
\hline 25.06 .07 & 36,49 & 72,94 & 9,0 & 146 & 6,2 & \\
\hline 25.06 .07 & 36,48 & 73,00 & 10,0 & 57 & 0,0 & $3,1( \pm 4,4)$ \\
\hline 10.10 .07 & 36,48 & 72,94 & 11,5 & 466 & 0,0 & \\
\hline 10.10 .07 & 36,48 & 72,96 & 11,8 & 2077 & 1,6 & \\
\hline 19.10 .07 & 36,48 & 72,95 & 11,0 & 13 & 7,7 & \\
\hline 19.10.07 & 36,48 & 72,94 & 11,0 & 15 & 13,3 & $5,7( \pm 6,1)$ \\
\hline 26.11 .07 & 36,48 & 72,94 & 14,0 & 35 & 0,0 & 0,0 \\
\hline 11.12 .07 & 36,36 & 72,90 & 11,0 & 87 & 0,0 & \\
\hline 11.12 .07 & 36,47 & 72,94 & 12,0 & 629 & 11,1 & $5,6( \pm 7,8)$ \\
\hline Total & & & & 3525 & 3,3 & \\
\hline
\end{tabular}


invierno y primavera del año 2007 a bordo de la embarcación 'Kay Kay II' de la Universidad de Concepción. La obtención de las muestras se realizó con redes tipo Bongo (300 $\mu \mathrm{m}$ trama, $60 \mathrm{~cm}$ diámetro boca, equipada con flujómetro) mediante arrastres oblicuos con una duración menor a $10 \mathrm{~min}$. La velocidad de arrastre fue menor a 2 nudos ya que los huevos son susceptibles al estrés mecánico provocado por la red (Cameron et al. 1992). En cada muestreo se monitoreó la temperatura superficial del mar debido a que esta característica hidrográfica es un inductor reconocido de anormalidades (Wiegand et al. 1989, Aritaki et al. 1996). Para los meses de noviembre y diciembre se registraron las características oceanográficas con CTD (modelo SBE 19plus).

Las muestras de plancton fueron fijadas a bordo en formalina neutralizada al 5\% y luego observadas en el laboratorio con microscopio estereoscópico donde se separaron los huevos de S. bentincki. La identificación se realizó en base a los caracteres señalados por Herrera et al. (1987). Los huevos fueron clasificados por estadio de desarrollo embrionario según la secuencia de once estadios descrita por Sepúlveda et al. (2000). Los huevos que presentaban alguna desviación atípica en la morfología embrionaria normal (Fig. 1a-o) fueron considerados anormales, los cuales fueron fotografiados y se analizaron con mayor aumento (45X) bajo lupa estereomicroscópica (Nikon E200) para asignarles estadios y realizar su descripción detallada.

Para descartar posibles alteraciones morfológicas producidas por la exposición al fijador, huevos vivos sin anormalidades obtenidos durante el año 2008 se fotografiaron antes y después de ser fijados en formalina al 5\%. Sin embargo, como las abundancias de huevos durante el 2008 fueron bajas, esta actividad sólo se pudo realizar con algunos estadios.
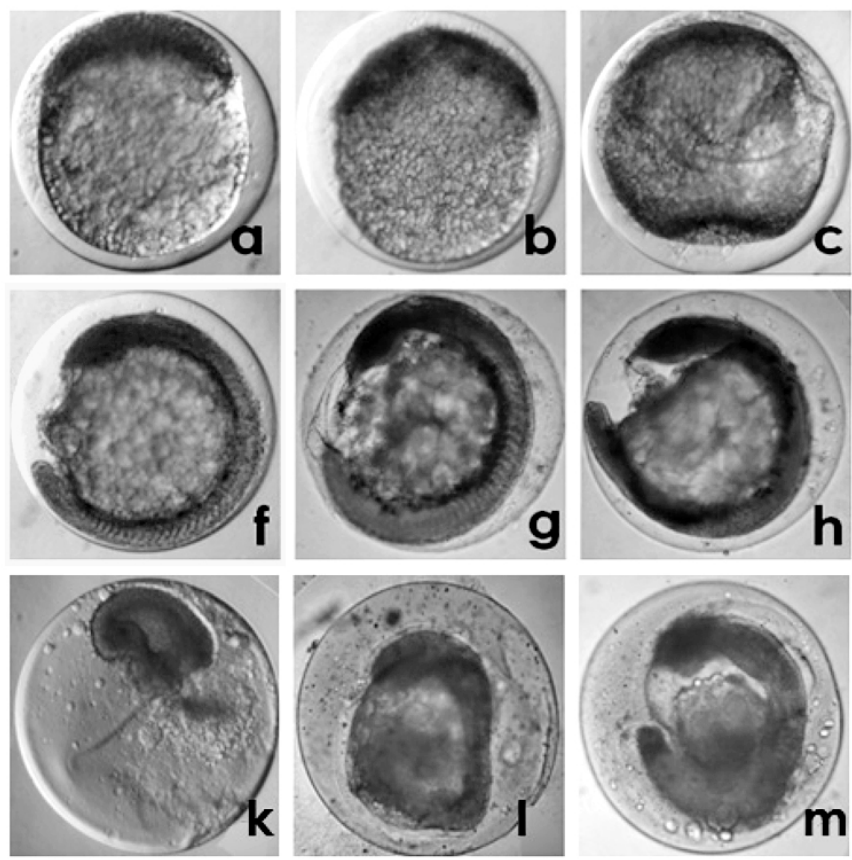
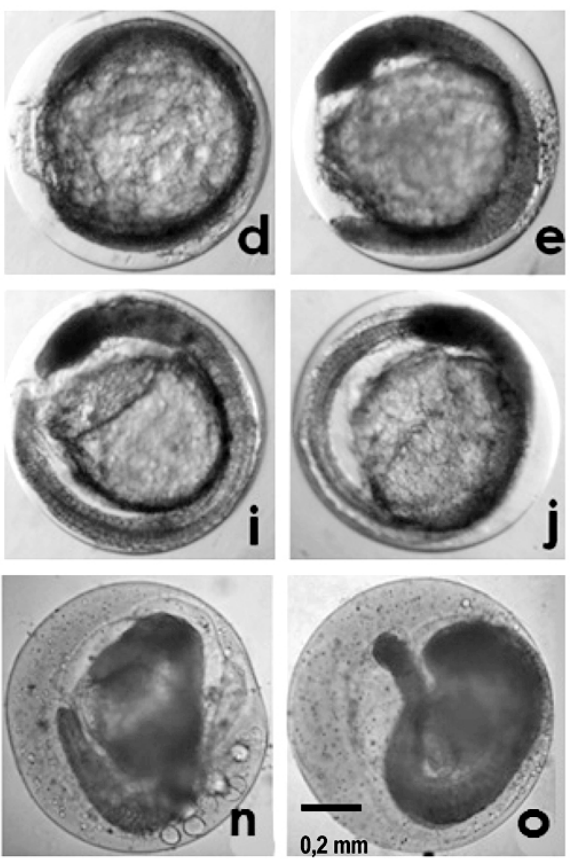

Figura 1

Strangomera bentincki: (a-j) huevos normales y (k-o) anormales en diferentes estadios de desarrollo embrionario observados en muestras fijadas en formalina neutralizada (5\%). a: estadio II, b: estadio III, c: estadio IV, d: estadio V, e: estadio VI,

f: estadio VII, g: estadio VIII, h: estadio IX, i: estadio X, j: estadio XI, k: estadio IV con detención en el avance del anillo embrionario, l-o: notocorda torcida en distintos estadios de desarrollo

Strangomera bentincki: (a-j) Normal and (k-o) abnormal eggs in the different developmental stages observed in samples preserved in buffered formalin (5\%). a: stage II, b: stage III, c: stage IV, d: stage V, e: stage VI, f: stage VII, g: stage VIII, h: stage IX, $\mathrm{i}$ : stage $\mathrm{X}$, j: stage XI, k: stage IV with detention in germinal ring advance, 1-o: embryonic axis 
El número de huevos de sardina analizados por muestreo correspondió a la abundancia total de estos en la muestra. En cada fecha de muestreo, se separó la muestra por estadio de desarrollo embrionario y se contabilizó en cada uno de ellos el número de huevos normales y anormales. Adicionalmente, en aquellos meses con mayor nivel de anormalidades se midió la totalidad de los huevos anormales y una submuestra de huevos normales. Para esto se fotografió la totalidad de los huevos y se estimó el diámetro mayor con la ayuda del software ImageJ (Rasband 1997-2008). A partir de esta medida se calculó el volumen del huevo $\left(\mathrm{mm}^{3}\right)$ asumiendo la forma de una esfera $\left(4 / 3 \pi r^{3}\right.$, donde $r$ es el radio).

Para evaluar la existencia de diferencias en la frecuencia de anormalidades a lo largo de la época reproductiva y en las distintas etapas del desarrollo embrionario, los datos fueron agrupados por meses de muestreo y por estadio de desarrollo. Se utilizó el estadístico de contingencia $\chi^{2}$ con el programa PAST (Hammer et al. 2001). Para evaluar la relación entre la frecuencia de anormalidades y la temperatura superficial del mar en el momento de muestreo se calculó el coeficiente de correlación de Pearson con el programa STATISTICA (StatSoft Inc. 2004). No se consideró el resto de las variables oceanográficas ya que no se contó con el registro adecuado en todos los muestreos. Para evaluar diferencias de tamaño entre huevos normales y anormales se aplicó una prueba $t$ con STATISTICA.

\section{Resultados}

Del total de muestras analizadas fue posible observar embriones desde el estadio IV en adelante, es decir, desde el momento en que el blastodisco abarca más de un tercio del corion. En todos estos estadios fue posible constatar algún grado de anormalidad, excepto en el estadio V (Fig. 1d).

En embriones normales en estadio IV, el blastodisco tiene un grado de desarrollo que abarca más de un tercio de la masa vitelina, la cavidad de segmentación ya formada alcanza gran tamaño y se ubica en un costado del huevo (Fig 1c). En huevos anormales, el blastodisco se presenta como una proliferación atípica de células, con forma de bulto, en el ápice de la masa vitelina, el avance del anillo germinal se detiene, lo que provoca la interrupción del desarrollo del embrión (Fig. 1k).

En el estadio VI, el embrión, que se aprecia claramente, aún no comienza a separarse de la masa de vitelo, a pesar de que abarca más de la mitad del corion. Los somitos se observan excepto en la región caudal; se inicia la diferenciación de los ojos (Fig. 1e). En huevos anormales se puede apreciar principalmente el eje corporal torcido (Fig. 11), lo que puede ir acompañado de la disgregación del vitelo.

Entre los estadios VII y XI, la distinción entre estadios se logra por el avance del grado de desprendimiento de la cola en relación con la masa de vitelo (Fig. 1f-j). Fue esta relación la que permitió en aquellos embriones anormales que presentaban el eje corporal torcido, la asignación del respectivo estadio de desarrollo (Fig. 1mo).

Desde las muestras de plancton vivas, sólo fue posible encontrar huevos en los estadios IV, VI y VIII. Estos fueron fotografiados vivos y fijados en formalina al $5 \%$. La observación detallada demostró que no hubo una alteración en la morfología del embrión provocada por la exposición al fijador y que erróneamente fuese considerada como anomalía. La formalina sólo provocó que el tejido del embrión se oscureciera y una contracción de la masa vitelina, cambios que no son considerados anormalidades. En este análisis se tomó la precaución de utilizar los estadios más sensibles a la aparición de anormalidades (estadios IV, VI y VIII).

\section{Cuantificación de anormalidades durante el desarrollo embrionario}

En total se analizaron 3525 huevos provenientes de nueve muestras obtenidas en cuatro meses de la época reproductiva de $S$. bentincki. De este total, un 3,3\% correspondió a embriones con algún tipo de anomalía (Tabla 1).

En los diferentes estadios del desarrollo embrionario de S. bentincki, el porcentaje de anormalidades varió significativamente (prueba $\chi^{2}, P<0,001 ; \mathrm{n}=10$ ). En general, a medida que el desarrollo embrionario avanza hay una disminución en el nivel de anormalidades encontradas (Fig. 2a).

El estadio que presentó más anormalidades fue el IV, en el cual se detiene el avance del anillo germinal, provocando que no se concrete el cierre del blastoporo (Fig. 1k). Luego, los estadios con más frecuencia de anormalidades fueron el VII y VIII, etapas en las que se produce entre otros procesos, la elongación del extremo caudal (Fig. 11-m). El máximo de anormalidades se produce durante la gastrulación, mientras que el resto de anormalidades se produce durante el proceso de organogénesis (Fig. 2a).

El nivel de anormalidades en los meses analizados presentó diferencias entre sí (prueba $\chi^{2}, P<0,001$, n= 4). Los meses en los que se presentó un mayor porcentaje de anormalidades correspondieron a octubre y diciembre 
(Tabla 1). Se pudo apreciar una leve tendencia a un aumento en el nivel de anormalidades a medida que la estación reproductiva avanzó.

Durante noviembre y diciembre, el registro de CTD mostró en las estaciones muestreadas que las condiciones oceanográficas entre los 0 y $15 \mathrm{~m}$ de profundidad, fluctuaron entre los $9,8-12,3^{\circ} \mathrm{C}, 32,4-34,5$ ups y $6,1-6,4$ $\mathrm{mg} \mathrm{L}^{-1}$ oxígeno disuelto. El nivel de anormalidades a lo largo del gradiente de temperatura superficial que se presentó durante los meses evaluados fue variable, no encontrándose una relación entre el nivel de anormalidades detectado y la temperatura superficial del mar en el momento de muestreo $(r=-0,174, P=0,653)$.

Se detectaron diferencias significativas de tamaño entre huevos normales y anormales (prueba $t, P<0,001$, $\mathrm{n}_{\text {normales }}=596 \mathrm{n}_{\text {anormales }}=109$ ), siendo de menor tamaño los huevos anormales. Esta tendencia se mantuvo al agrupar por estadio (Fig. 2b, estadio IV-VI prueba $t, P<0,001$, $\mathrm{n}_{\text {normales }}=212 \mathrm{n}_{\text {anormales }}=79$; estadio $>$ VI prueba $t, P<0,001$, $\mathrm{n}_{\text {normales }}=210 \mathrm{n}_{\text {anormales }}=30$ ).
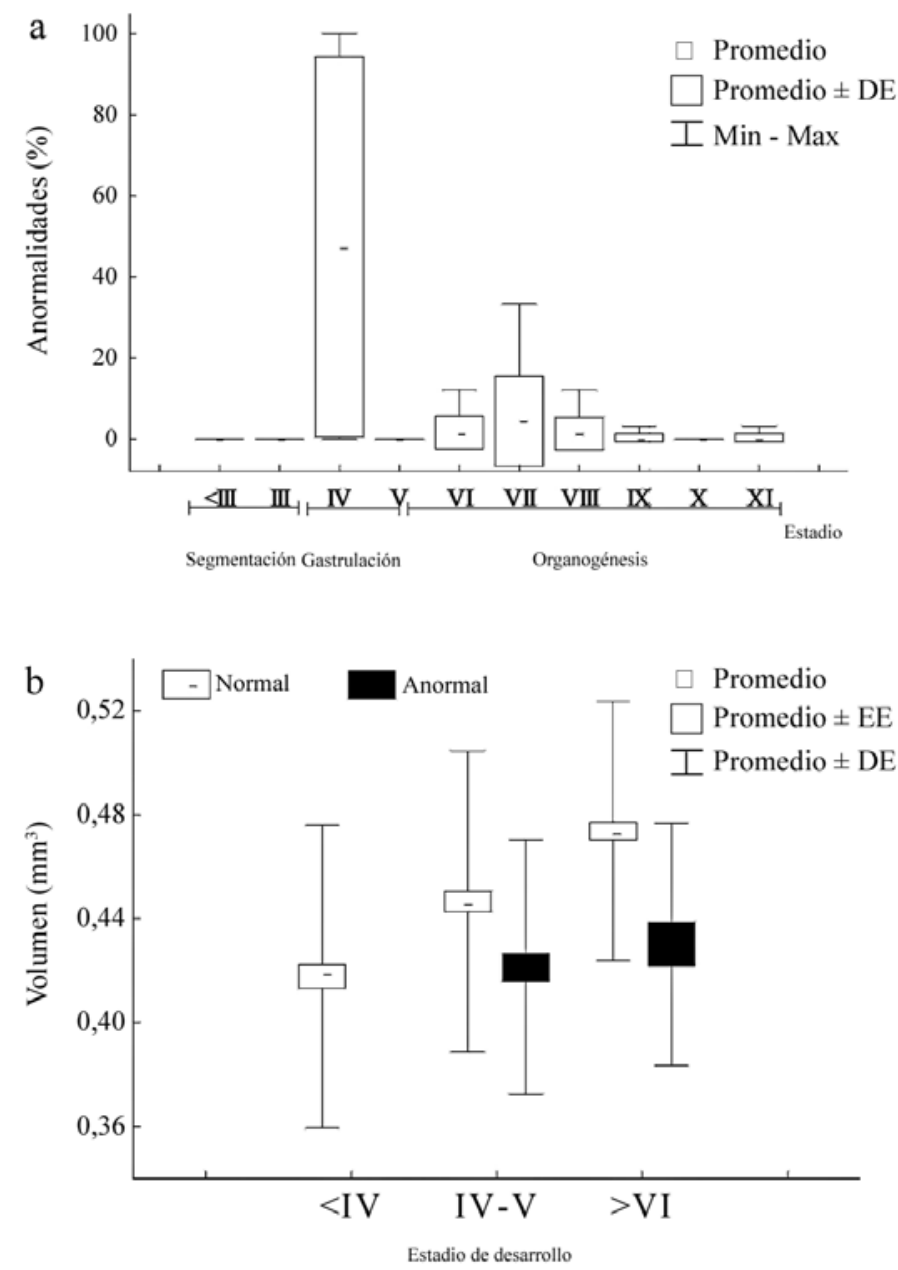

Figura 2

a) Anormalidades morfológicas (\%) en los distintos estadios de desarrollo embrionario de Strangomera bentincki; los valores corresponden a todos los muestreos combinados. b) Variación en los volúmenes de huevo normales y anormales de Strangomera bentincki agrupados por estadios de desarrollo

a) Morphological abnormalities (\%) at the different embryonic developmental stages in Strangomera bentincki; values correspond to all sampling dates pooled. b) Volume variations in normal and abnormal

Strangomera bentincki eggs, pooled by developmental stage 


\section{Discusión}

En esta investigación se determinó para la sardina común, Strangomera bentincki, el tipo de anormalidades presentes en forma natural en las distintas etapas del desarrollo embrionario y su frecuencia a lo largo de la estación reproductiva, en el área de desove costera de la zona centro sur de Chile, durante el año 2007. Las anormalidades detectadas en el desarrollo embrionario de la sardina común, en general, coinciden con las encontradas en embriones de otras especies de teleósteos. Estas alteraciones en el desarrollo son observables mediante inducción experimental, ya sea frente a contaminantes o factores abióticos (Tabla 2), como también en condiciones naturales (Makhotin et al. 2001), y se presentan en varios ordenes disímiles de teleósteos tales como Clupeiformes, Gadiformes, Cipriniformes, Ciprinidontiformes y Pleuronectiformes.

Entre las anormalidades comunes en distintas especies de peces, destacan el cierre irregular del blastoporo provocado por la detención del avance del anillo germinal y la torsión notocordal, especialmente en la región caudal (Tabla 2). Estas alteraciones corresponderían a errores durante la epibolía, fase en la cual se establecen y yuxtaponen las primeras tres capas germinales. En este proceso fallaría la correspondencia entre movimientos morfogenéticos relativamente independientes, a través de los cuales se constituye la región axial del embrión incluyendo el tronco y la cola (Makhotin et al. 2001, Kunz 2004, Ali 2007). Otra fase clave en el desarrollo de peces corresponde a la segmentación temprana, en la cual se observan alteraciones en la morfología de los blastómeros (Avery \& Brown 2005, Avery et al. 2009). En el caso de la sardina común, el desove ocurre durante noche y el muestreo se hace durante la madrugada, razón por la cual no se encontraron embriones en fase de desarrollo previos a la gastrulación, por lo que no fue posible su observación.

En los embriones de sardina común se pudo cuantificar una incidencia decreciente de anormalidades a medida que el desarrollo embrionario avanza. La gastrulación es la etapa con mayor frecuencia de anormalidades, las que durante la organogénesis disminuyen. Esta tendencia ya ha sido descrita para varias especies de peces (Cameron et al. 1992) y se explicaría porque los estadios tempranos, previos a la organogénesis, reflejan la condición parental. Es decir, las variaciones en las características fisiológicas y reproductivas del adulto repercuten en la calidad de sus huevos (Schreck et al. 2001, Hershberger et al. 2005). La alta tasa de mortalidad diaria natural de huevos registrados para esta especie (0,8-0,98 día $\left.{ }^{-1}\right)$ (Cubillos et al. 2007), sugiere que estas anormalidades podrían ser

\section{Tabla 2}

\section{Anormalidades en el desarrollo embrionario reportadas por diversos autores en distintas especies de peces}

Embryonic developmental anomalies reported by several authors in different fish species

\begin{tabular}{|c|c|c|c|c|}
\hline Especie & Anormalidades & Estadio $(*)$ & Causas & Autor \\
\hline Cyprinus carpio & Torsión notocordal & VII & $\begin{array}{c}\text { Exposición a metales } \\
\text { pesados } \\
\text { (cobre o plomo) }\end{array}$ & $\begin{array}{c}\text { Jezierska et al. } \\
\text { (2000). }\end{array}$ \\
\hline Fundulus heteroclitus & $\begin{array}{l}\text { (1) Alteración en desarrollo del embrión por escaso } \\
\text { desarrollo de la región cefálica (2) Embrión sin partes } \\
\text { del cuerpo distinguibles, se observa por encima del } \\
\text { vitelo }\end{array}$ & VI-XI & $\begin{array}{c}\text { Exposición a } \\
\text { metiltestosterona }\end{array}$ & $\begin{array}{l}\text { Boudreau et al. } \\
\text { (2005). }\end{array}$ \\
\hline Engraulis anchoita & Torsión notocordal, especialmente en región caudal & VII-XI & $\begin{array}{l}\text { Temperatura } 19-20^{\circ} \mathrm{C} \text {. } \\
\text { Salinidad } 3,5-8,4 \text { ups }\end{array}$ & $\begin{array}{c}\text { De Ciechomski } \\
\text { (1966). }\end{array}$ \\
\hline $\begin{array}{l}\text { Buglossidium luteum } \\
\text { Hipoglossoides platessoides } \\
\text { Limanda limanda } \\
\text { Phrynorhombus norwegicus } \\
\text { Microstomus kitt } \\
\text { Platichthys flesus } \\
\text { Pleuronectes platessa } \\
\text { Psetta máxima } \\
\text { Solea solea }\end{array}$ & $\begin{array}{l}\text { Agregados celulares atípicos con concentración plasmática } \\
\text { defectuosa. } \\
\text { Segmentación irregular de los blastómeros } \\
\text { Falla en cierre de blastoporo. Afloramientos como ampollas } \\
\text { desde el eje corporal } \\
\text { (1) Notocordos torcidos y/o deformaciones de la región } \\
\text { cefálica (2) Alteración en tamaño de ojos y anormalidades } \\
\text { en la pigmentación }\end{array}$ & $\begin{array}{c}\text { II-VI } \\
\text { III } \\
\text { IV-V } \\
\text { VII-XI }\end{array}$ & $\begin{array}{c}\text { Exposición a metales } \\
\text { pesados } \\
\text { (cobre, arsénico y zinc) e } \\
\text { hidrocarburos clorinados }\end{array}$ & $\begin{array}{c}\text { Cameron et al. } \\
\text { (1992). }\end{array}$ \\
\hline
\end{tabular}


producidas por factores endógenos, relacionados con la condición parental.

En la anchoveta Engraulis ringens, otro Clupeiforme presente en la misma zona geográfica, se ha observado una variación durante la estación reproductiva tanto en la calidad bioquímica como en el tamaño del huevo, disminuyendo ambos a medida que la época de desove avanza (Castro et al. 2002, Llanos-Rivera \& Castro 2004). Estas características del huevo influyen directamente en la supervivencia de los estadios larvales tempranos (Castro et al. 2009). Para Strangomera bentincki, en el presente estudio se observó una tendencia no significativa al aumento en el nivel de anormalidades morfológicas embrionarias a medida que la estación reproductiva avanza. Esto puede resultar relevante dadas las similitudes en las características reproductivas que la sardina comparte con E. ringens, sugiriendo que se podrían atribuir a efectos de la calidad de los huevos. Lo anterior adquiere relevancia si se considera que en este estudio se constató que los huevos anormales eran significativamente de menor tamaño que los normales.

El nivel de anormalidades detectado para la totalidad de los huevos analizados varió entre 3-13\%, y concuerda con lo cuantificado para otras especies de clupeidos: Anchoviella sp. (3-8\%), Sardina pilchardus (12\%) y Engraulis enchrasicolus (9\%) (Klumpp et al. 2002, Klumpp \& Von Westernhagen 1995, Yannopoulos \& Yannopoulos 1981, respectivamente). Estos niveles son similares a los observados en otros teleósteos como Limanda limanda, Pleuronectes platessa, especies en que el porcentaje de anormalidades varía entre 4 y $8 \%$ en ambientes sin o con escasa contaminación (Klumpp \& Von Westernhagen 1995). El área de muestreo considerada en nuestro estudio ha sido catalogada como un área con escasa contaminación (Palma et al. 2007). De la misma manera, los valores de temperatura superficial del mar observados en nuestro estudio se encuentran dentro del rango característico de la época de desove de esta especie, la que oscila entre $10^{\circ} \mathrm{C}$ y $17^{\circ} \mathrm{C}$ (Sepúlveda et al. 2000).

La información proporcionada en este estudio valida el uso de muestras fijadas en formalina neutralizada al 5\%, incluso en los estadios tempranos más sensibles. Sin embargo, las estimaciones futuras podrían complementarse con observaciones a partir de embriones vivos. El presente estudio demuestra que la sardina común, $S$. bentincki, es una buena especie de estudio para cuantificar fluctuaciones en el nivel de anormalidades embrionarias. Esta especie, además de ser endémica, tiene gran importancia comercial y presenta un amplio rango de distribución a lo largo de las costas de Chile. Los niveles de anormalidades detectados pueden servir de referencia para futuros monitoreos en el área, ya sean estudios de calidad ambiental, de fluctuaciones en las condiciones hidrográficas, así como para estudios que busquen determinar variaciones en viabilidad de estados tempranos debido a efectos maternales en condiciones naturales.

\section{Agradecimientos}

Esta investigación fue financiada por el Programa de Investigación Marina de Excelencia (PIMEX-Nueva Aldea) Universidad de Concepción - CFI-Nueva Aldea. Los autores agradecen por la colaboración en cruceros, separación de muestras de ictioplancton y montaje de experimentos a S. Soto, A. Yánez, M.I. Muñoz, P. Barrientos y J. Méndez, del Laboratorio de Oceanografía Pesquera y Ecología Larval (LOPEL) de la Universidad de Concepción, y especialmente a S. Soto por su ayuda en el manejo del programa ImageJ.

\section{Literatura citada}

Ali N. 2007. Teratology in zebrafish embryos: a tool for risk assessment. Report. Master of Science. Programme in veterinary medicine for international students. Faculty of Veterinary, Medicine and Animal Science, Swedish University of Agricultural Sciences, Report 65: 1-68.

Aritaki M, T Seikai \& M Kobayasi. 1996. Reduction of morphological abnormalities in brown sole by larval rearing with higher temperature and early feeding of Artemia nauplii. Nippon Suisan Gakkaishi 62: 857-864.

Avery T \& J Brown. 2005. Investigating the relationship among abnormal patterns of cell cleavage, egg mortality and early larval condition in Limanda ferruginea. Journal of Fish Biology 67: 890-896.

Avery T, S Killen \& T Hollinger. 2009. The relationship of embryonic development, mortality, hatching success, and larval quality to normal or abnormal early embryonic cleavage in Atlantic cod, Gadus morhua. Aquaculture 289: 265-273.

Boudreau M, S Courtnay, D MacLatchy, C Berube, H Hewitt \& G Van der Kraak. 2005. Morphological abnormalities during early life development of the estuarine mummichog, Fundulus heteroclitus, as an indicator of androgenic and anti-androgenic endocrine disruption. Aquatic Toxicology 71: 357-369.

Cameron P, J Berg, V Dethlefsen \& $\mathbf{H}$ von Westernhagen. 1992. Developmental defects in pelagic embryos of several flatfish species in the southern North Sea. Netherlands Journal of Sea Research 29: 239-256.

Castro L, A Llanos, J Blanco, E Tarifeño, R Escribano \& M Landaeta. 2002. Influence of latitudinal variations in spawning habitat characteristics on the early life history 
traits of the anchoveta, Engraulis ringens, off northern and central Chile. GLOBEC Report 16: 42-45.

Castro L, G Claramunt, M Krautz, A Llanos-Rivera \& $\mathbf{P}$ Moreno. 2009. Egg trait variation in anchoveta Engraulis ringens: a maternal response to changing environmental conditions in contrasting spawning habitats. Marine Ecology Progress Series 381: 237-248.

Cubillos L, M Canales, D Bucarey, A Rojas \& R Alarcón. 1999. Época reproductiva y talla media de primera madurez sexual de Strangomera bentincki y Engraulis ringens en el período 1993-1997, en la zona centro-sur de Chile. Investigaciones Marinas 27: 73-85.

Cubillos L, D Bucarey \& M Canales. 2002. Monthly abundance estimation for common sardine Strangomera bentincki and anchovy Engraulis ringens in the centralsouth Chile (34-40 S). Fisheries Research 57: 117-130.

Cubillos L, P Ruiz, G Claramunt, S Gacitúa, S Núñez, L Castro, K Riquelme, C Alarcón, C Oyarzún \& A Sepúlveda. 2007. Spawning, daily egg production, and spawning stock biomass estimation for common sardine (Strangomera bentincki) and anchovy (Engraulis ringens) off central southern Chile in 2002. Fisheries Research 86: 228-240.

Córdova G \& F Balbontín. 2006. Distribución espacial de la abundancia y de la talla de ocho tipos de larvas de peces entre la Boca del Guafo y Bahía Anna Pink, Zona Austral de Chile. Ciencia y Tecnología del Mar 29(1): 153-161.

De Ciechomski J. 1966. Influence of some environmental factors upon the embryonic development of the argentine anchovy Engraulis anchoita (Hubbs and Marini). CALCOFI Reports 11: 67-71.

Fischer W. 1958. Huevos, crías y primeras prelarvas de la "anchoveta" (Engraulis ringens) Jenyns. Revista de Biología Marina 8(1, 2 y 3): 111-124.

Hammer Ø, D Harper \& P Ryan. 2001. PAST: Paleontological Statistics software package for education and data analysis. Paleontologia Electronica 4(1): 1-9.

Herrera G, E Tarifeño \& M Orellana. 1987. Descripción de huevos y primeras fases larvales de la sardina común (Strangomera bentincki) y del machuelo (Ethmidium maculatum). Biología Pesquera 16: 107-113.

Hershberger P, N Elder, J Wittouck, K Stick \& R Kocan. 2005. Abnormalities in larvae from the once-largest Pacific herring population in Washington State result primarily from factors independent of spawning location. Transactions of the American Fisheries Society 134: 326 337.

Jezierska B, K Eugowska, M Witeska \& P Sarnowski. 2000. Malformations of newly hatched common carp larvae. Electronic Journal of Polish Agricultural Universities 3(2). [en línea] <http://www.ejpau.media.pl/volume3/issue2/ fisheries/art-01.html>
Kjorsvik E, A Mangor-Jensen \& I Holmefjord. 1990. Egg quality in fishes. Advances in Marine Biology 26: 71-113.

Klumpp D \& H Von Westernhagen. 1995. Biological effects of pollutants in Australian tropical coastal waters: Embryonic malformations and chromosomal aberrations in developing fish eggs. Marine Pollution Bulletin 30: 158165 .

Klumpp D, C Humphrey, H Huasheng \& F Tao. 2002. Toxic contaminants and their biological effects in coastal waters of Xiamen, China. II. Biomarkers and embryo malformation rates as indicators of pollution stress in fish. Marine Pollution Bulletin 44: 761-769.

Kunz Y. 2004. Developmental biology of teleost fishes, 636 pp. Fish \& Fisheries Series, Springer, Dordrecht.

Llanos-Rivera A \& LR Castro. 2004. Latitudinal and seasonal egg size variation of the anchoveta Engraulis ringens, off the Chilean coast. Fishery Bulletin 102: 207-212.

Makhotin V, P Solemdal, K Korsbrekke \& A Salthaug. 2001. Types and frequency of malformations and mortality in eggs ofArcto-Norwegian cod: a field study. ICES CM2001/N:12: $1-17$.

Mellinger J. 1995. Malformations embryonnaires et larvaires dues à la pollution marine. Mise au point bibliographique. Ichtyophysiologica Acta 18: 145-152.

Moser H \& E Ahlstrom. 1985. Staging anchovy eggs. En: Lasker R (ed). An egg production method for estimating spawning biomasss of pelagic fish: application to the northern anchovy (Engraulis mordax). NOAA Technical Report NMFS 36: 37-41.

Palma M, F González, H Romo, E Ruiz \& C Fuentealba. 2007. Efectos de la contaminación sobre la diversidad genética de Mazzaella laminarioides (Bory) Fredericq (Gigartinales, Rhodophyta) en bahías. Gayana Botánica 64: 24-32.

Rasband W. 1997-2008. ImageJ 1.34s National Institutes of Health USA [en línea] <http://rsb.info.nih.gov/ij/>

Schreck C, W Contreras-Sanchez \& M Fitzpatrick. 2001. Effects of stress on fish reproduction, gamete quality, and progeny. Aquaculture 197: 3-24.

Sepúlveda A, L Cubillos, S Núñez, T Canales, D Bucarey \& ARojas. 2000. Antecedentes biológicos del stock desovante de anchoveta y sardina común de la $\mathrm{V}$ y IX regiones. Informe Final Projecto FIP 97-04: 1-200. [en-línea] < http:/ /www.fip.cl/prog_recurso/1997/9704.htm>

SERNAPESCA. 2008. Anuario Estadístico de Pesca 2007, 140 pp. Ministerio de Economía, Fomento y Reconstrucción, Servicio Nacional de Pesca, Valparaíso.

Serra R, M Aguayo, O Rojas, J Cañón \& F Inostroza. 1979. Anchoveta Engraulis ringens (Jenyns) y sardina común Clupea (Strangomera) bentincki. En: CORFO/IFOP (ed). Estado actual de las principales pesquerías nacionales. Bases para un desarrollo pesquero, $52 \mathrm{pp}$. Instituto de Fomento Pesquero, Valparaíso. 
Stachel SE, D Grunwald \& PZ Myers. 1993. Lithium perturbation and goosecoid expression identify a dorsal specification pathway in the pregastrula zebrafish. Development 117: 1261-1274.

StatSoft Inc. 2004. STATISTICA (data analysis software system), version 7. [en línea] <http://www.statsoft.com>

Von Westernhagen H. 1988. Sublethal effects of pollutants on fish eggs and larvae. En: Hoar W \& DJ Randall (eds). The physiology of developing fish. Fish Physiology 2: 253346. Academic Press, New York.
Von Westernhagen H, H Dethlefsen \& M Haarich. 2001. Can a pollution event be detected using a single biological effects monitoring method? Marine Pollution Bulletin 42: 294-297.

Wiegand M, J Hataley, C Kitchen \& L Buchanan. 1989. Induction of developmental abnormalities in larval goldfish, Carassius auratus L., under cool incubations. Journal of Fish Biology 35: 85-95.

Yannopoulos A \& C Yannopoulos. 1981. Fish egg mortality and abnormal embryogenesis. Rapports et Proces \& Verbaux des Reunions Commission Internationale pour I' Exploration de la Mer 27:143-146.

Recibido el 31 de agosto de 2009 y aceptado el 31 de diciembre de 2009 